\title{
Cognitive processing of spatial relations in Euclidean diagrams
}

\author{
Yacin Hamami ${ }^{\mathrm{a}, *}$, Milan N.A. van der Kuil ${ }^{\mathrm{b}}$, John Mumma ${ }^{\mathrm{c}}$, Ineke J.M. van der Ham ${ }^{\mathrm{b}}$ \\ ${ }^{a}$ Centre for Logic and Philosophy of Science, Vrije Universiteit Brussel, Brussels, Belgium \\ ${ }^{\mathrm{b}}$ Department Health, Medical and Neuropsychology, Leiden University, Leiden, the Netherlands \\ ${ }^{\mathrm{c}}$ Philosophy Department, California State University of San Bernardino, San Bernardino, CA, USA
}

\section{A R T I C L E I N F O}

\section{Keywords:}

Euclidean diagrams

Spatial relation processing

Metric and topological relations

Exact and co-exact relations

Coordinate and categorical relations

\begin{abstract}
A B S T R A C T
The cognitive processing of spatial relations in Euclidean diagrams is central to the diagram-based geometric practice of Euclid's Elements. In this study, we investigate this processing through two dichotomies among spatial relations-metric vs topological and exact vs co-exact-introduced by Manders in his seminal epistemological analysis of Euclid's geometric practice. To this end, we carried out a two-part experiment where participants were asked to judge spatial relations in Euclidean diagrams in a visual half field task design. In the first part, we tested whether the processing of metric vs topological relations yielded the same hemispheric specialization as the processing of coordinate vs categorical relations. In the second part, we investigated the specific performance patterns for the processing of five pairs of exact/co-exact relations, where stimuli for the co-exact relations were divided into three categories depending on their distance from the exact case. Regarding the processing of metric vs topological relations, hemispheric differences were found for only a few of the stimuli used, which may indicate that other processing mechanisms might be at play. Regarding the processing of exact vs co-exact relations, results show that the level of agreement among participants in judging co-exact relations decreases with the distance from the exact case, and this for the five pairs of exact/co-exact relations tested. The philosophical implications of these empirical findings for the epistemological analysis of Euclid's diagram-based geometric practice are spelled out and discussed.
\end{abstract}

\section{Introduction}

Anyone who has ever encountered a geometry book or paper, or attended a geometry course or seminar, knows that diagrams are ubiquitous to geometric practice, from the most elementary to the most advanced level. The diagram-based geometric practice that has received the most attention is undoubtedly that of Euclid's Elements (Euclid, 1959)—-the classical text which for over two millennia played a foundational role for all of mathematics. This practice has been investigated from the perspective of various fields, with fundamental contributions from the history of mathematics (Mueller, 1981; Netz, 1999), the philosophy of mathematics (Manders, 2008), and the education of mathematics (Hartshorne, 2000; Hartshorne, 2000b). Whatever the perspective adopted, however, it must be recognized that at the heart of any diagram-based geometric practice lies a cognitive agent interacting with diagrams-i.e., with external representations of a visuo-spatial nature-and that an account of this interaction is in order if we are to understand the functioning of these practices-a point recently emphasized by Giaquinto (2007) and Ferreirós (2015) in the so-called philosophy of mathematical practice (Mancosu, 2008). Yet, although the cognitive role of external representations in mathematics has been investigated empirically in the particular cases of algebra (Landy \& Goldstone, 2007) and calculation (Landy \& Goldstone, 2010), interaction with diagrams in geometric practice has received little to no attention from experimental psychology (for a notable exception, see Koedinger and Anderson (1990)). The present study aims to progress in this latter direction by addressing the archetypical case of the diagrambased geometric practice of Euclid's Elements.

Central to the use of diagrams in Euclid's geometric practice is the processing of spatial relations in Euclidean diagrams. The most famous example of this phenomenon occurs in the very first proof of the Elements-i.e., the proof of proposition 1 from book I, which establishes that an equilateral triangle can be constructed on any given line segment-where Euclid famously reads off from the diagram that the two circles intersect, thus introducing the resulting intersection point in the course of his proof (see Fig. 1). According to the received 20th century view, Euclid's use of diagrams in proofs is symptomatic of an unrigorous approach to geometry. The seminal analysis of Euclid's diagram-based geometric practice developed by Manders (2008) has revealed, however, that such reading off from diagrams in Euclid's geometric

\footnotetext{
* Corresponding author at: Pleinlaan 2, B-1050 Brussels, Belgium.

E-mail address: yacin.hamami@gmail.com (Y. Hamami).
} 


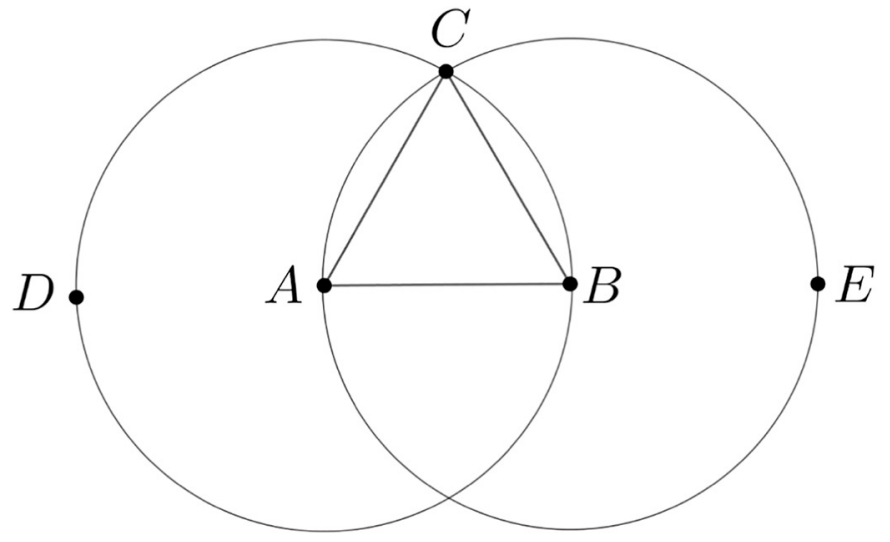

Fig. 1. This is the Euclidean diagram accompanying proposition 1 from Book I of Euclid's Elements. The geometrical proof of this proposition runs as follows: "Let $A B$ be the given finite straight line. Thus it is required to construct an equilateral triangle on the straight line $A B$. With centre $A$ and distance $A B$ let the circle $B C D$ be described; again, with centre $B$ and distance $B A$ let the circle $A C E$ be described; and from the point $C$, in which the circles cut one another, to the points $A, B$ let the straight lines $C A, C B$ be joined. Now, since the point $A$ is the centre of the circle $C D B, A C$ is equal to $A B$. Again, since the point $B$ is the centre of the circle $C A E, B C$ is equal to $B A$. But $C A$ was also proved equal to $A B$; therefore each of the straight lines $C A, C B$ is equal to $A B$. And things which are equal to the same thing are also equal to one another; therefore $C A$ is also equal to $C B$. Therefore the three straight lines $C A, A B, B C$ are equal to one another. Therefore the triangle $A B C$ is equilateral; and it has been constructed on the given finite straight line $A B$. (Being) what it was required to do." (Euclid, 1959, pp. 241-242).

reasoning always proceeds in a highly controlled way. Manders has shown, specifically, that only a certain category of spatial relations is actually read off from the diagram in Euclid's proofs. This category is characterized in terms of two dichotomies among spatial relations in Euclidean diagrams: metric vs topological and exact vs co-exact.

The metric vs topological dichotomy is based on the appearance or topology of a Euclidean diagram, which is defined by Manders as consisting of "the inclusions and contiguities of regions, segments, and points in the diagram" (Manders, 2008, p. 89). This leads to an equivalence relation between Euclidean diagrams, namely that of having the same appearance. Metric and topological relations can then be defined as follows: a spatial relation between two or more objects is topological if it can be characterized as an equivalence class, or a union of equivalence classes, of diagrams in the space of all possible diagrams containing exactly those objects; a spatial relation between two or more objects is metric if it is not topological. Typical examples of topological relations are a point being within a circle (inclusion), or a line being tangent to a circle (contiguity). Typical examples of metric relations are the equality and inequality of length between two line segments, or the congruence between two figures-such relations being too fine-grained, so to speak, to be characterized as an equivalence class, or a union of equivalence classes, of diagrams having the same appearance. ${ }^{1}$

The exact vs co-exact dichotomy is due to Manders and plays a pivotal role in his account of Euclid's diagram-based geometric practice. A spatial relation between two or more objects is co-exact if, for every possible diagram containing exactly those objects, if the relation holds in the diagram, then it must be unaffected by some range of every

\footnotetext{
${ }^{1}$ It should be noted that, in a wider sense, every spatial relation in a Euclidean diagram is metric, insofar as it can always be defined in metric terms. In this sense, topological relations would also count as metric relations, and there would not be a dichotomy between metric and topological relations. The term metric has, however, been used in a narrower sense in the literature on Euclidean diagrams to refer to a finer class of spatial relations that could not be defined in terms of appearance of Euclidean diagrams.
}

continuous variation of it. A spatial relation between two or more objects is exact if, for every possible diagram containing exactly those objects, there exists a continuous variation of the diagram for which the relation obtains only in isolated cases. Typical examples of co-exact relations are the intersection between two curves-as in the example of proposition 1 from Book I of the Elements discussed above, where the intersection between the two circles is unaffected by any reasonable deformation of the two circles-or the inequality of length between two line segments. Typical examples of exact relations are a line being tangent to a circle or the equality of length between two line segments-such relations being immediately affected by any deformation, however small, of a diagram instantiating them. Table 1 provides a nonexhaustive list of spatial relations in Euclidean diagrams classified in terms of the two dichotomies metric vs topological and exact vs coexact.

The key insight of Manders' analysis is that Euclid only reads off from the diagram spatial relations that are both topological and co-exact. Interestingly, the reason advanced by Manders as to why Euclid only reads off co-exact relations is arguably cognitive in nature. First of all, Manders points out that, a mathematical practice being inherently a social endeavor, its success and cohesion requires a high-level of agreement among its participants, the absence of which leading to disruption or dissolution of the practice-what Manders calls 'disarray' (Manders, 2008, p. 82). For diagram-based geometric practices such as the one of Euclid's Elements, this means in particular that the practice ought to assure uniformity of responses in reading off spatial relations from geometric diagrams. Because exact relations only obtain in isolated cases, and because the concrete diagrams produced in practice are always to some extent imperfect, judging exact relations from concrete diagrams is bound to yield divergent responses from the participants, thus failing the demand for uniformity of responses. Co-exact relations, on the other hand, because they are stable under a range of variations of how a concrete diagram may be produced, can be judged from concrete diagrams without leading to disagreements among participants, provided that the level of imperfection of the concrete diagrams produced in practice remains 'reasonable'. This explanation-that we shall refer to as Manders' rationale - is cognitive in nature insofar as it concerns primarily the way by which spatial relations are judged from concrete diagrams.

The representation and processing of spatial relations is an important topic of research for several fields of cognitive science. In cognitive psychology, researchers have investigated the mental representations of spatial relations (e.g., Bryant \& Tversky, 1999; McNamara, 1986; Stevens \& Coupe, 1978), the use of spatial relations in cognitive maps (e.g., Kuipers, 1982; Kuipers, 1983; Tversky, 1993), and various forms of reasoning with spatial relations (e.g., Byrne \& Johnson-Laird, 1989; Knauff, 2013). In linguistics, research has focused on spatial prepositions (Coventry \& Garrod, 2004; Landau \& Jackendoff, 1993; Retz-Schmidt, 1988) such as 'part of' (Winston, Chaffin, \& Herrmann, 1987), 'in' and 'on' (McDonough, Choi, \& Mandler, 2003), or 'in the front/back of' and 'on the left/right of' (Franklin, Tversky, \& Coon, 1992), which have been investigated through empirical studies (e.g., Hayward \& Tarr, 1995; Munnich, Landau, \& Dosher, 2001) and the development of computational models (e.g., Kelleher \& Costello, 2009). In artificial intelligence, researchers have been concerned, among other things, with the design of programs capable of learning and recognizing spatial relations in visual scenes (e.g., Winston, 1970), the construction of mathematical models of how humans encode spatial relations in pictures (e.g., Freeman, 1975), the formal representation of topological spatial relations (e.g., Egenhofer \& Franzosa, 1991), and the development of formal calculi for representing and reasoning about regions and their connections (e.g., Cohn \& Hazarika, 2001; Randell, Cui, \& Cohn, 1992).

To our knowledge, the exact/co-exact distinction has not been investigated empirically. On the other hand, the metric/topological distinction is very similar to a distinction originally introduced by Kosslyn 
Table 1

A non-exhaustive list of spatial relations in Euclidean diagrams.

\begin{tabular}{|c|c|c|}
\hline & Exact & Co-exact \\
\hline \multirow[t]{7}{*}{ Metric } & $\begin{array}{l}\text { Two line segments: equality of } \\
\text { length }\end{array}$ & $\begin{array}{l}\text { Two line segments: inequality of } \\
\text { length }\end{array}$ \\
\hline & $\begin{array}{l}\text { Two angles: equality of } \\
\text { magnitude }\end{array}$ & $\begin{array}{l}\text { Two angles: inequality of } \\
\text { magnitude }\end{array}$ \\
\hline & $\begin{array}{l}\text { Two areas: equality of } \\
\text { magnitude }\end{array}$ & Two areas: inequality of magnitude \\
\hline & Two figures: congruence & Two figures: non-congruence \\
\hline & $\begin{array}{l}\text { Two pairs of line segments: } \\
\text { proportionality }\end{array}$ & $\begin{array}{l}\text { Two pairs of line segments: non- } \\
\text { proportionality }\end{array}$ \\
\hline & Four points: concyclicity & Four points: non-concyclicity \\
\hline & Two lines: parallel & Two lines: non-parallel \\
\hline \multirow[t]{6}{*}{ Topological } & $\begin{array}{l}\text { Three lines: intersection in } a \\
\text { common point }\end{array}$ & $\begin{array}{l}\text { Three lines: pairwise intersection } \\
\text { in three distinct points }\end{array}$ \\
\hline & $\begin{array}{l}\text { Three curves: intersection in a } \\
\text { common point }\end{array}$ & $\begin{array}{l}\text { Three curves: pairwise intersection } \\
\text { in three distinct points }\end{array}$ \\
\hline & A line, a curve: tangency & A line, a curve: non-tangency \\
\hline & Two curves: tangency & Two curves: non-tangency \\
\hline & A point, a segment: on & A point, a segment: within \\
\hline & A point, a region: on & A point, a region: within \\
\hline
\end{tabular}

Note: This is a non-exhaustive list of spatial relations in Euclidean diagrams classified in terms of the two dichotomies metric vs topological and exact vs coexact. For further examples of spatial relations in Euclidean diagrams, see (Manders, 2008, pp. 92-93).

(1987) between coordinate and categorical spatial relations which has received considerable attention in experimental psychology. Coordinate spatial relations reflect metric, absolute relations between objects or an object and the observer. These relations are used in motor actions. In the act of picking up a glass, for instance, the coordinate relations between the hand and the glass are continuously updated. Categorical spatial relations concern abstract, propositional relations between objects or an object and the observer. Categorical relations are typically used in communication, for instance when giving directions 'take a left at the train station' (Kosslyn, 1987; Kosslyn et al., 1989). A long line of experimental work supports the view that there is a clear dichotomy between categorical and coordinate information processing, most clearly reflected by a pattern of hemispheric lateralization. Coordinate information is preferentially processed by the right hemisphere, whereas categorical information is processed with a left hemisphere bias (for a review, see van der Ham, Postma, and Laeng (2014)).

In this study, we investigate the cognitive processing of spatial relations in Euclidean diagrams through the metric vs topological and exact vs co-exact dichotomies. With respect to the former, the main issue is whether metric and topological relations are processed differently by the two brain hemispheres, that is, whether the processing of metric vs topological relations yields the same hemispheric specialization as the processing of coordinate vs categorical relations. With respect to the latter, the main issues are whether judging exact relations in Euclidean diagrams does lead to disagreements among different individuals, while judging co-exact relations does not, and whether such a pattern can be observed equally for metric and topological relations alike.

The experiment presented below aims to tackle these different issues. The experiment is composed of two parts. In the first part, the
Table 3

The exact/co-exact relations tested in the second part of the study.

\begin{tabular}{lll}
\hline & Exact & Co-exact \\
\hline \multirow{2}{*}{ Metric } & Two line segments: equality of & Two line segments: inequality of \\
& length & length \\
& Two angles: equality of & Two angles: inequality of \\
& magnitude & magnitude \\
& Two lines: parallel & Two lines: non-parallel \\
Topological & Three lines: intersection in a & Three lines: pairwise intersection \\
& common point & in three distinct points \\
& A line, a circle: tangency & A line, a circle: non-tangency \\
\hline
\end{tabular}

processing of metric and topological relations between four different sets of geometric objects was investigated (see Table 2). We shall say that the relations among a given set of geometric objects constitute a relation type, which is then characterized by the considered set of geometric objects. For each relation type, the processing of metric and topological relations was tested in dedicated series of trials. For addressing the main issue relative to the processing of metric vs topological relations, we used the classical visual half field methodology in which, for each trial, stimuli are presented very briefly to one of the two visual half fields, providing input to the contralateral hemisphere. This allows for the detection of potential hemispheric biases at a behavioral level. Given the multitude of findings concerning lateralization of spatial relations (van der Ham et al., 2014), we expect to observe a left hemispheric/right visual field advantage for the processing of topological relations, and a right hemispheric/left visual field advantage for the processing of metric relations, following previous results on the processing of coordinate vs categorical relations. In the second part, the processing of five pairs of exact/co-exact relations-among which three were metric and two were topological-was investigated (see Table 3). Each pair was tested in a dedicated series of trials. Within each trial, participants were asked to judge which of the two considered relations holds in a specific Euclidean diagram composed of exactly the objects involved in the relations. For addressing the main issues relative to the processing of exact vs co-exact relations, we divided the stimuli for each pair of exact/co-exact relations into four classes: one class where the exact relation holds, and three different classes where the co-exact relation holds and which correspond to three decreasing levels of variation from the exact case, that is, three decreasing distances from the exact case. We expect to observe that the closer a class of co-exact stimuli is from the exact case, the greater the disagreement among participants will be in judging relations from stimuli of this class. Furthermore, we expect to observe this pattern for metric and topological relations alike. In this second part of the experiment, we also used the classical visual half field methodology in order to investigate eventual hemispheric specializations for the metric vs topological relations tested.

This study aims thus to combine the philosophical analysis of Euclid's diagram-based geometric practice developed by Manders with the experimental methodology of the field of spatial relation processing to investigate one of the central components of the interaction with diagrams in elementary geometry, namely the cognitive processing of spatial relations in geometric diagrams.

Table 2

The metric/topological relations tested in the first part of the study.

\begin{tabular}{|c|c|c|}
\hline & Metric & Topological \\
\hline A point, a circle & Length of the line segment connecting the point to the center of the circle & Inside, on, outside \\
\hline Two points, a line & Magnitude of the angle formed by the two line segments connecting each point to the middle of the line & Same side, opposite side \\
\hline Three points & Closer to the left point, closer to the right point & Between, non-between \\
\hline Two lines & Parallel, non-parallel & Intersection, non-intersection \\
\hline
\end{tabular}




\section{Method}

\subsection{Participants}

A total of 25 participants took part in the experiment, one of whom reported visual problems with one eye and was therefore excluded from the final dataset. Therefore the data of 24 participants was included in the analyses ( 12 male, 12 female, mean age $=24.5, \mathrm{SD}=4.5$ ). One female participant did not complete the second part of the experiment, and so only her data for the first part of the experiment were included in the analyses. All participants were right-handed and had normal or corrected-to-normal vision. The experiment was performed according to the Declaration of Helsinki (2013) and all participants provided informed consent prior to the experiment.

\subsection{Tasks and stimuli}

The experiment consisted of two parts: the first aimed at assessing hemispheric lateralization patterns for the processing of metric vs topological relations; the second aimed at assessing particular performances for the processing of exact vs co-exact relations.

\subsubsection{First part: processing of metric $v$ s topological relations}

To be able to assess performance and lateralization patterns for the processing of metric vs topological relations, we tested the processing of metric and topological relations for the four relation types in Table 2 in a visual half field task design. Metric and topological relations for each relation type were tested in dedicated series of trials. Descriptions of the stimuli used are presented in Figs. 2 and 3. For each relation type, both a metric and a topological instruction were used.

Stimuli for the relation types PointCircle (Fig. 2a) and 2PointsLine

(Fig. 2b) were presented in a match-to-sample design, to assess lateralization within working memory. In this design, participants were asked to compare two consecutively presented stimuli. The trials consisted of a central fixation cross $(500 \mathrm{~ms})$, central presentation of stimulus 1 (150 ms), retention interval (duration $1000 \mathrm{~ms}$ ), central fixation cross $(500 \mathrm{~ms})$, lateral presentation of stimulus $2(150 \mathrm{~ms}, 2.5$ degrees of visual angle), a response window set at a maximum of $2000 \mathrm{~ms}$. All stimuli were presented $2.94 \mathrm{~cm}$ to the left or right of the center of the screen. Stimuli size varied by type (see Fig. 2) but never exceeded a width and height of $3.97 \mathrm{~cm}$. Stimuli for the relation type PointCircle consisted of a combination of a circle, a point, and a line connecting the point to the center of the circle. The point was placed inside the circle $(0.56$ or $0.85 \mathrm{~cm}$ from the center), on the circle $(1.14 \mathrm{~cm}$ from the center), or outside the circle $(1.43$ or $1.72 \mathrm{~cm}$ from the center). The metric instruction was to decide whether or not the line segments in the two consecutive stimuli were of equal length. The topological instruction was to decide whether or not the point was within the same spatial category in the two consecutive stimuli, that is, either inside the circle, on the circle, or outside the circle. Stimuli for the relation type 2PointsLine (Fig. 2b) consisted of a combination of a horizontal line, two points, and two line segments connecting each of the two points to the middle of the horizontal line. The magnitude of the angle between the line segments ranged from 15 to 180 degrees. The metric instruction was to decide whether or not the magnitude of the angle formed by the two line segments was equal in the two consecutive stimuli, whereas the topological instruction was to decide whether the two points were on the same side or on opposite sides of the horizontal line.

Perceptual trials with single stimulus presentation were used for the relation types 3Points (Fig. 3a) and 2Lines (Fig. 3b). These trials consisted of a central fixation cross $(500 \mathrm{~ms})$, lateral stimulus presentation

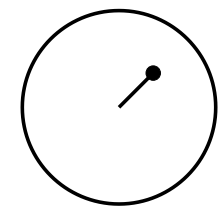

Inside

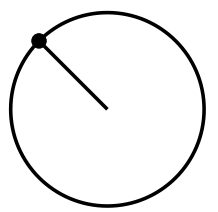

On

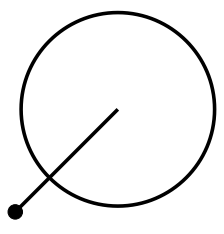

Outside

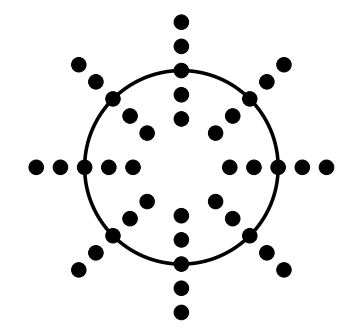

a
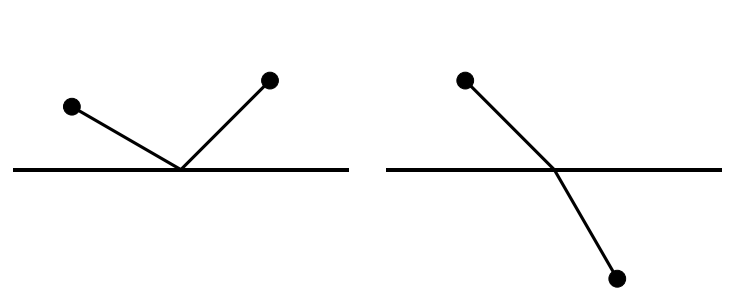

Same side
Opposite sides

b

Fig. 2. a. Examples of stimuli for the relation type PointCircle. The picture on the right depicts all possible positions for the point. In the metric case, a "match" response should be given when the length of the segment is equal in the two stimuli. In the topological case, a "match" response should be given when the point is either inside, on, or outside in the two stimuli.

$b$. Examples of stimuli for the relation type 2PointsLine. The picture on the right depicts all possible positions for the points. In the metric case, a "match" response should be given when the magnitude of the angle formed by the two segments is equal in the two stimuli. In the topological case, a "match" response should be given when the two points are either on the same side or on opposite sides of the line in the two stimuli. 

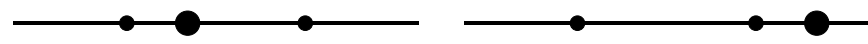

Between

a
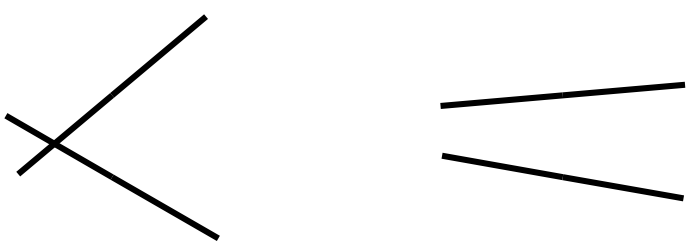

Intersection

b

Fig. 3. a. Examples of stimuli for the relation type 3Points. In the metric case, the instruction was to decide whether the larger point was closer to the left or to the right smaller point. In the topological case, the instruction was to decide whether or not the larger point was between the two smaller points.

$b$. Examples of stimuli for the relation type 2Lines. In the metric case, the instruction was to decide whether or not the two lines were parallel. In the topological case, the instruction was to decide whether or not the two lines intersect.

(150 ms, 2.5 degrees of visual angle), and a response window of maximally $2000 \mathrm{~ms}$. All stimuli were presented $2.94 \mathrm{~cm}$ to the left or right of the center of the screen. Stimuli size varied by type (Fig. 3) but never exceeded a width and height of $3.97 \mathrm{~cm}$. The stimuli for the relation type 3Points consisted of a horizontal line, with two smaller points and a larger point. The metric instruction was to decide whether the larger point was closer to the left or to the right smaller point. The topological instruction was to decide whether or not the larger point was between the two smaller points. The stimuli for the relation type 2Lines consisted of two lines that were either parallel or have a relative angle of 5 to 60 degrees. The metric instruction was to decide whether or not the two lines were parallel, whereas the topological instruction was to decide whether or not the two lines intersect.

\subsubsection{Second part: processing of exact $v$ s co-exact relations}

To be able to compare performances for the processing of exact vs co-exact relations, stimuli for the five relation types of Table 3 were used in a visual half field task design. The processing of exact and coexact relations for each relation type was tested in a dedicated series of trials. Examples of stimuli for each relation type are presented in Fig. 4. For each relation type-each consisting of a pair of an exact and a coexact relation-the stimuli were divided into four classes: one class where the exact relation holds, and three different classes where the coexact relation holds and which correspond to three decreasing distances (far, medium, close) from the exact case (see Fig. 4). The instruction always took the form of asking whether the exact relation holds in the presented stimulus.

Stimuli for all relation types were presented in perceptual trials with single stimulus presentation. These trials consisted of a central fixation cross $(500 \mathrm{~ms})$, lateral stimulus presentation $(150 \mathrm{~ms}, 2.5$ degrees of visual angle), and a response window of maximally $2000 \mathrm{~ms}$. Stimuli were presented $2.94 \mathrm{~cm}$ to the left or right of the center of the screen. Stimuli had a maximum width and height of $3.97 \mathrm{~cm}$. Stimuli for the relation type 2Linesegments (Fig. 4a) consisted of two line segments presented simultaneously. The instruction was to decide whether or not the length of the two line segments were equal. If the line segments were not equal in length, they differed either of 1.43 (far), 0.69 (medium), or $0.48 \mathrm{~cm}$ (close). The stimuli for the relation type 2Angles (Fig. 4b) consisted of two angles presented simultaneously. Here the instruction was to decide whether or not the magnitudes of the two angles were equal. If the angles were not equal in magnitude, they differed either of 60 (far), 40 (medium), or 20 degrees (close). Stimuli for the relation type 2Lines (Fig. 4c) consisted of two lines presented simultaneously, of which the participant was asked to decide whether or not they were parallel. If not, they differed of 15 (far), 10 (medium), or 5 degrees (close). The stimuli for the relation type LineCircle (Fig. 4d) consisted of a combination of a line and a circle. The instruction was to decide whether or not the line was tangent to the circle. If the line was not tangent to the circle, then the line was 0.32 (far), 0.21 (medium), or $0.11 \mathrm{~cm}$ (close) 'away' from tangency. Stimuli for the relation type 3Lines (Fig. 4e) consisted of three lines which did or did not intersect in a common point. Participants were asked to decide whether or not the three lines were intersecting in a common point. If the three lines did not intersect in a common point, the deviant line was 0.32 (far), 0.21 (medium) or $0.11 \mathrm{~cm}$ (close) 'away' from the intersection point of the two lines forming the central cross.

For both parts of the experiment, a total of 48 trials were presented for each relation type, 24 to the left visual field, 24 to the right visual field, equally divided over the response options. Both accuracy and response times were registered. Trials with response times below $200 \mathrm{~ms}$ and above $2000 \mathrm{~ms}$ were excluded. Trials presented to the left visual field (LVF)—respectively to the right visual field (RVF)—were interpreted as reflecting right hemisphere ( $\mathrm{RH}$ ) processing-respectively left hemisphere (LH) processing. Mean performance scores were calculated for each participant for each trial combination of stimulus type and visual field.

\subsection{Procedure}

Participants first received a brief description of the experiment and were asked to sign the informed consent. Task order was randomized for each participant in a single session. Participants were seated centrally in front of the computer screen $(1920 \times 1080)$, using a chin rest, at a distance of $68.7 \mathrm{~cm}$. Each task was introduced by the experimenter and was preceded by multiple examples. The experimenter ensured the participant understood the instructions before starting the actual measurements. The participant responded to each trial using the keyboard and pressing a button with their right index or middle finger, depending on the response option. Accuracy and response times were collected for each trial.

\subsection{Statistical analysis}

For the first part of the experiment, lateralization patterns were assessed. For each relation type, a repeated measures general linear model (GLM) was performed, including visual field (LVF, RVF) and spatial relation (metric, topological) as within subject factors. Any significant interactions of visual field and spatial relation were followed up by post hoc comparisons.

In the second part of the experiment, first lateralization patterns were assessed with a paired sample $t$-tests, comparing visual fields (LVF, RVF) for each relation type. Next, a comparison was performed between the exact and co-exact trials by means of paired sample t-tests, followed by a comparison of the three co-exact conditions (far, medium, close) disregarding visual field, in a repeated measures GLM. Any significant effect of the co-exact conditions was followed up by post hoc comparisons.

All analyses were performed for both accuracy and response times separately. 

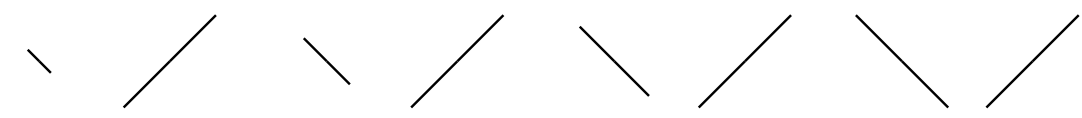

Co-exact (far)

Co-exact (medium)

Co-exact (close)

Exact

a
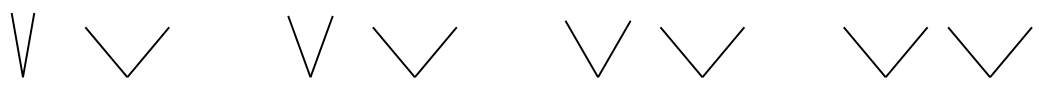

Co-exact (far)

Co-exact (medium)

Co-exact (close)

Exact

b

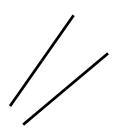

Co-exact (far)

$\mathrm{c}$

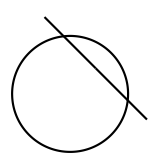

Co-exact (far) d

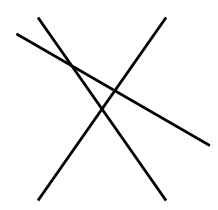

Co-exact (far)

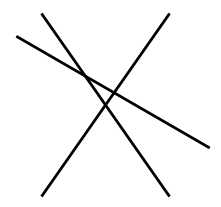

Co-exact (medium)

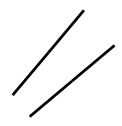

Co-exact (medium)

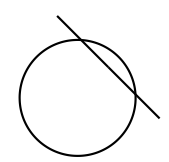

Co-exact (medium)

Co-exact (close)

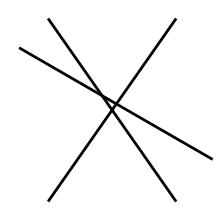

Co-exact (close)

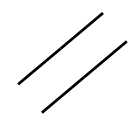

Exact

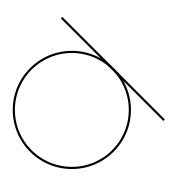

Exact

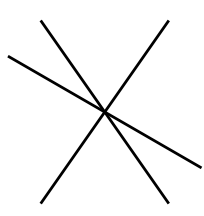

Exact
Fig. 4. $a$. Examples of stimuli for the relation type 2Linesegments. The stimuli in the co-exact conditions far, medium, and close differed with respect to the difference of length between the two line segments; the orientation as well as the length of the two line segments were varied within each condition. In the exact condition, the stimuli depict two line segments of equal length; the orientation as well as the length of the two line segments were varied. The instruction was to decide whether or not the lengths of the two line segments were equal.

$b$. Examples of stimuli for the relation type 2Angles. The stimuli in the co-exact conditions far, medium, and close differed with respect to the difference of magnitude between the two angles; the orientation as well as the magnitude of the two angles were varied within each condition. In the exact condition, the stimuli depict two angles of equal magnitude; the orientation as well as the magnitude of the two angles were varied. The instruction was to decide whether or not the magnitude of the two angles were equal. c. Examples of stimuli for the relation type 2Lines. The stimuli in the co-exact conditions far, medium, and close differed with respect to the relative orientation between the two lines; the orientation of the two lines were varied within each condition. In the exact condition, the stimuli depict two parallel lines; the orientation of the two lines were varied. The instruction was to decide whether or not the two lines were parallel.

$d$. Examples of stimuli for the relation type LineCircle. The stimuli in the co-exact conditions far, medium, and close differed with respect to the distance between the line and the periphery of the circle; the orientation of the line was varied within each condition. In the exact condition, the stimuli depict the line tangent to the circle; the orientation of the line was varied. The instruction was to decide whether or not the line was tangent to the circle.

$e$. Examples of stimuli for the relation type 3Lines. The stimuli in the co-exact conditions far, medium, and close differed with respect to the distance between the center of the cross formed by two lines (which was always kept fixed) and the third line; the orientation of the third line was varied within each condition. In the exact condition, the stimuli depict the three lines intersecting in a common point; the orientation of the third line was varied. The instruction was to decide whether or not the three lines were intersecting in a common point. e

\section{Results}

\subsection{First part: processing of metric vs topological relations}

To assess the lateralization patterns a repeated measures GLM including visual field (LVF, RVF) and spatial relation (metric, topological) was performed, for both accuracy and response times, for each relation type. For one participant, performance for relation type 2Lines was substantially below chance level $(<10 \%)$ and therefore excluded. Mean performances for each relation type are reported in Tables $4 \mathrm{a}$ (metric case) and 4b (topological case).

For the relation type PointCircle, analysis of accuracy showed a significant main effect of spatial relation, $\mathrm{F}(1,23)=152.10, p<.001$, partial eta squared $=0.869$, indicating that performance was more accurate for the topological than metric trials. No further significant effects were found for either accuracy or response times. For the relation type 2PointsLine also a main effect of spatial relation was found for accuracy, $F(1,23)=54.82, p<.001$, partial eta squared $=0.839$, again with more accurate responses for topological trials, compared to metric trials. No further significant effects were found for accuracy or
Table 4a

Mean scores for accuracy (\%) and response times (ms) for left and right visual field presentations of stimuli in the metric relation case (first part of the experiment).

\begin{tabular}{|c|c|c|c|c|c|c|c|}
\hline & \multicolumn{2}{|l|}{ Left } & \multicolumn{2}{|l|}{ Right } & \multirow[b]{2}{*}{$\mathrm{t}$} & \multirow[b]{2}{*}{$\mathrm{df}$} & \multirow[b]{2}{*}{$\mathrm{p}$} \\
\hline & M & $\mathrm{SD}$ & M & SD & & & \\
\hline \multicolumn{8}{|l|}{ Accuracy (\%) } \\
\hline PointCircle & 70.4 & 8.76 & 67.94 & 8.77 & 1.02 & 23 & n.s. \\
\hline 2PointsLine & 80.38 & 9.09 & 82.29 & 7.9 & -0.69 & 23 & $\mathrm{n} . \mathrm{s}$ \\
\hline 3Points & 94.19 & 6.33 & 94.01 & 7.04 & 0.13 & 23 & $\mathrm{n} . \mathrm{s}$ \\
\hline 2Lines & 94.4 & 8.82 & 94.52 & 7.79 & -0.09 & 23 & $\mathrm{n} . \mathrm{s}$ \\
\hline \multicolumn{8}{|c|}{ Response time (ms) } \\
\hline PointCircle & 718.16 & 203 & 729.2 & 191.9 & -0.55 & 23 & n.s \\
\hline 2PointsLine & 697.5 & 159.79 & 720.05 & 157.8 & -1.72 & 23 & 0.09 \\
\hline 3Points & 502.3 & 140.83 & 499.34 & 148.17 & 0.33 & 23 & n.s. \\
\hline 2Lines & 460.64 & 103.79 & 475.6 & 114.94 & -1.29 & 23 & n.s. \\
\hline
\end{tabular}

response times. For the relation type 3Points, also a main effect of spatial relation was found for accuracy, $\mathrm{F}(1,23)=54.82, \mathrm{p}<.001$, partial eta squared $=0.704$. Here performance was more accurate for 
Table 4b

Mean scores for accuracy (\%) and response times (ms) for left and right visual field presentations of stimuli in the topological relation case (first part of the experiment).

\begin{tabular}{|c|c|c|c|c|c|c|c|}
\hline & \multicolumn{2}{|l|}{ Left } & \multicolumn{2}{|l|}{ Right } & \multirow[b]{2}{*}{$\mathrm{t}$} & \multirow[b]{2}{*}{ df } & \multirow[b]{2}{*}{$\mathrm{p}$} \\
\hline & M & SD & M & SD & & & \\
\hline \multicolumn{8}{|l|}{ Accuracy (\%) } \\
\hline PointCircle & 88.24 & 8.81 & 88.58 & 9.66 & -0.19 & 23 & n.s. \\
\hline 2PointsLine & 93.51 & 6.77 & 93.53 & 6.51 & -0.01 & 23 & n.s. \\
\hline 3Points & 80.52 & 9.56 & 78.96 & 10.12 & 0.71 & 23 & n.s. \\
\hline 2Lines & 94.89 & 4.88 & 95.52 & 4.05 & -0.67 & 22 & n.s. \\
\hline \multicolumn{8}{|l|}{$\begin{array}{l}\text { Response time } \\
\quad \text { (ms) }\end{array}$} \\
\hline PointCircle & 760.68 & 140.12 & 742.58 & 147.11 & 1.37 & 23 & n.s. \\
\hline 2PointsLine & 684.28 & 144.98 & 695.36 & 134.88 & -0.88 & 23 & n.s. \\
\hline 3Points & 671.64 & 150.19 & 707.46 & 143.58 & -2.84 & 23 & $>0.01$ \\
\hline 2Lines & 473.46 & 98.57 & 467.55 & 104.86 & 1.06 & 22 & n.s. \\
\hline
\end{tabular}

the metric trials, in comparison to the topological trials. For accuracy, no further significant effects were found. For response times, a main effect of spatial relation was found, $\mathrm{F}(1,23)=43.48, \mathrm{p}<.001$, partial eta squared $=0.654$, as well as a main effect of $\mathrm{VF}, \mathrm{F}(1,23)=4.70$, $p<.041$, partial eta squared $=0.170$. The interaction of spatial relation and VF was also significant, $\mathrm{F}(1,23)=5.95, \mathrm{p}<.05$, partial eta squared $=0.206$. This indicates that responses were faster for metric compared to topological trials, and for LVF/RH compared to RVF/LH trials. Furthermore, a significant effect for topological trials was found in particular, with an advantage for LVF/RH trials, compared to RVF/ LH trials $(p=.009)$, no VF effect was found for metric trials. For the relation type 2Lines, no significant effects were found for either accuracy or response times.

\subsection{Second part: processing of exact vs co-exact relations}

Paired sample $t$-tests were performed between left and right VF for each relation type and for both accuracy and response times. This resulted in 2 significant differences in VF. For the relation type 2Angles (equality of magnitude), LVF/RH showed a lower response time than the RVF/LH, t $(22)=2.84, p<.01$. For the relation type 3Lines (intersection in a common point), performance for LVF/RH was significantly more accurate than for RVF/LH, t $(22)=2.10, p<.05$. Furthermore, two lateralization effects were at trend level, response times for the relation type 2Linesegments (equality of length) were marginally faster for the RVF/LH compared to the LVF/RH, $t$ $(22)=1.83, p=.081$, and an opposite pattern was found for the relation type LineCircle (tangency), $\mathrm{t}(22)=1.98, p=.060$, were the LVF/RH was marginally faster than the RVF/LH. Mean performances for each relation type are reported in Table 5.

Paired sample $t$-tests were performed between the exact and coexact trials for each relation type and for both accuracy and response times. A significant difference between exact and co-exact trials was found on two occasions. For the relation type 2Linesegments (equality of length), responses were faster for exact compared to co-exact trials, $t$ $(22)=2.59, p<.05$. Responses were more accurate for co-exact compared to exact trials for the relation type 3Lines (intersection in a common point), $\mathrm{t}(22)=2.33, p<.029$. Effects at trend level were found for the relation type 2Linesegments (equality of length), where responses were marginally more accurate for co-exact compared to exact trials, $\mathrm{t}(22)=2.00, p=.058$, and for the relation type LineCircle (tangency), where responses were faster for exact compared to co-exact trials, $\mathrm{t}(22)=1.80, p=.085$.

Effects on performance of the three co-exact conditions-corresponding to three decreasing distances from the exact case (far, medium, close)—were found for each relation type (see Fig. 5). With regard to accuracy, all general linear models of distances from the exact case showed a significant main effect of distance from the exact case ( $\mathrm{p}<.01$ in all cases). For the relation types 2Angles (equality of magnitude) and 3Lines (intersection in a common point), performance on medium distance trials was significantly lower than for far distance trials, and lower for close in comparison to medium. For the relation types 2Linesegments (equality of length), LineCircle (tangency), and 2Lines (parallel), performance for the far distance trials were significantly better in comparison to both medium and close ( $\mathrm{p}<.05$ in all cases). For response times, a highly similar data pattern was found. Again, distance from the exact case showed a significant main effect in all tasks ( $p \leq .001$ in all cases). For the relation types 2Linesegments (equality of length) and 3Lines (intersection in a common point), response times significantly increased from each distance to the next, and for the relation types 2Angles (equality of magnitude), LineCircle (tangency), and 2Lines (parallel), responses were significantly faster for the far distance trials in comparison to both medium and close distance trials.

Because the question asked-whether a certain relation holds in the presented stimulus-always admitted one of two possible answers, and because accuracy was above chance level for each stimulus, the accuracy measure for each stimulus reflected directly the level of agreement among participants in judging the spatial relation instantiated in the stimulus. More specifically, the accuracy measure reflected the percentage of participants constituting the largest group of participants who agreed in judging the considered relation, in which case $100 \%$ would correspond to the maximal level of agreement, and 50\% would correspond to the minimal level of agreement. It follows from this that the results reported for the accuracy measure translate directly to the level of agreement measure. In particular, this means that there is a significant main effect of the distance from the exact case on the level of agreement, that is, the level of agreement among participants in judging co-exact relations decreases with the distance from the exact case (see Fig. 5a). Furthermore, these effects were observed for metric and topological relations alike. These results are in line with our initial expectations regarding the processing of exact vs co-exact relations.

\section{Discussion}

The experiment just reported aimed to investigate the cognitive processing of spatial relations in Euclidean diagrams through two dichotomies that have been advanced as fundamental to understand how diagrams are used in the geometrical proofs of Euclid's Elements. The first part of the experiment was concerned with the processing of metric vs topological relations, while the second part was concerned with the processing of exact vs co-exact relations.

Regarding the processing of metric vs topological relations, we expected to observe a left hemisphere advantage for topological relations and a right hemisphere advantage for metric relation processing. Our results indicate that hemispheric differences exist for only a few of the stimuli used. The right hemisphere appears to be involved more for the relation type 2Angles (equality of magnitude), for which the precise magnitude of angles is to be assessed. This is in agreement with the typically found right hemisphere advantage for metric processing. In contrast, a right hemisphere advantage was also found for 3Points (between) and 3Lines (intersection in a common point), entailing topological decisions. The lack of lateralization effects and these two right hemispheric advantages may indicate that other processing mechanisms are at play. The layout of the stimuli may promote a large attentional scope, in order to take in all relevant metric information in the stimuli. As van der Ham et al. (2014) propose, such a large attentional scope is linked to a right hemispheric advantage and processing of coordinate spatial relations. In the current case, the layout of the stimuli may lead to such a right hemispheric advantage, as a large attentional scope likely helps in solving this task.

Regarding the processing of exact vs co-exact relations, we expected to observe that the closer a class of co-exact stimuli is from the exact 
Table 5

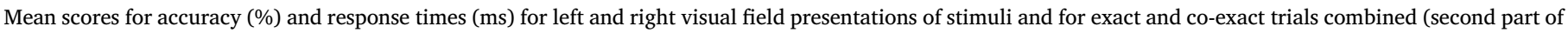
the experiment).

\begin{tabular}{|c|c|c|c|c|c|c|c|}
\hline & \multicolumn{2}{|l|}{ Left } & \multicolumn{2}{|l|}{ Right } & \multirow[b]{2}{*}{$\mathrm{t}$} & \multirow[b]{2}{*}{ df } & \multirow[b]{2}{*}{$\mathrm{p}$} \\
\hline & M & SD & M & $\mathrm{SD}$ & & & \\
\hline \multicolumn{8}{|l|}{ Accuracy (\%) } \\
\hline 2Linesegments & 89.67 & 6.75 & 87.85 & 6 & 1.09 & 23 & n.s \\
\hline 2Angles & 82.22 & 7.11 & 81.61 & 8.41 & 0.31 & 23 & n.s \\
\hline 2Lines & 96.07 & 4.5 & 93.77 & 5.44 & 1.69 & 23 & n.s \\
\hline LineCircle & 90.18 & 7.07 & 89.99 & 6.28 & 0.12 & 23 & n.s \\
\hline 3Lines & 77.05 & 9.66 & 70.87 & 11.64 & 2.10 & 23 & $<0.05$ \\
\hline \multicolumn{8}{|c|}{ Response time (ms) } \\
\hline 2Linesegments & 575.01 & 122.04 & 553.39 & 102.57 & 1.83 & 23 & 0.081 \\
\hline 2Angles & 586.62 & 136.31 & 621.34 & 153.58 & -2.84 & 23 & $<0.01$ \\
\hline 2Lines & 466.62 & 67.04 & 464.27 & 70.6 & 0.32 & 23 & n.s \\
\hline LineCircle & 485.39 & 75.79 & 502.84 & 74.74 & -1.98 & 23 & 0.060 \\
\hline 3Lines & 578.42 & 110.43 & 601.24 & 130.19 & -1.58 & 23 & n.s \\
\hline
\end{tabular}

case, the greater the disagreement among participants will be in judging relations from stimuli of this class. The results of the second part of the experiment support this hypothesis: for each of the five pairs of exact/co-exact relations tested, the level of agreement among participants in the three classes of co-exact stimuli decreases with the distance to the exact case. Furthermore, this effect was observed for both topological and metric relations alike. This means that, from a cognitive perspective, the exact/co-exact distinction is orthogonal to the metric/ topological distinction. The results also show that, for the five pairs of exact/co-exact relations, the level of agreement for the class of stimuli corresponding to the exact case was higher than the one for the class of co-exact stimuli closest to the exact case. This is not surprising, insofar as this reflects an intuitive expectation that chances to judge a co-exact stimulus that is very close to an exact one as instantiating an exact relation-e.g., judging that a line is tangent to a circle in a stimulus where the line is almost tangent to the circle-should be higher than the ones of judging an exact stimulus as instantiating a co-exact relation-e.g., judging that a line is not tangent to a circle in a stimulus where the line is indeed tangent to the circle.

These results have direct philosophical implications for two important themes of Manders' epistemological analysis of Euclid's diagram-based geometric practice.

First, they provide empirical support for Manders' rationale as to why Euclid only reads off co-exact relations from diagrams in the course of his geometrical proofs. To see this, imagine a given geometrical proof starting with a set of construction steps such that, for any geometric configuration that can be produced according to these steps, a certain exact relation $R$ always holds among some of its objects. Because any concrete diagram produced with ruler and compass is always imperfect to some extent, the concrete diagrams that can be produced according to these construction steps will always be such that the exact relation $R$ will never be perfectly instantiated. More specifically, in any such concrete diagram, the objects involved in $R$ will always come close to realizing $R$, the level of care with which the concrete diagram is produced determining how close. What our results show is that judging whether an exact relation $R$ holds in such diagrams is bound to yield a maximal level of disagreement among individuals. This means that if the diagram-based geometric practice of Euclid's Elements was to require of its participants to judge exact relations from Euclidean diagrams, it would not be able to maintain uniformity of responses in doing so, and the practice would then fall into disarray. Manders' $r a-$ tionale appears thus to be grounded, on the one hand, in the necessarily imperfect nature of concrete diagrams, and, on the other hand, in the cognitive difficulty of individuals to agree in judging exact relations in diagrams in which such relations are not perfectly instantiated.

Second, our results provide some empirical explanations for what Manders refers to as diagram discipline, that is, "standards for the proper production and refinement of diagrams" (Manders, 2008, p. 96). To see this, imagine a given geometrical proof starting with a set of construction steps such that, for any geometric configuration that can be produced according to these steps, a certain co-exact relation $R$ always holds among some of its objects. Some of the concrete diagrams produced according to these construction steps will be such that the relation $R$ will end up very close to the exact case. What our results show is that judging whether a co-exact relation holds in such diagrams is also bound to yield a maximal level of disagreement among individuals. These situations could then lead to what Manders calls 'disarray' (Manders, 2008, p. 82), that is, a disruption or dissolution of the practice caused by the absence of agreement among the practitioners as to which relations hold in the diagram. These situations can be avoided, however, by simply rejecting those diagrams for which uniformity of responses cannot be attained. This is precisely what diagram discipline consists in. More specifically, Manders emphasizes that "Diagram-based attribution requires [...] that the feature attributed appear in appropriately produced diagrams" (Manders, 2008, p. 96), and that when "defects are recognizable, and when they appear severe, or pertinent to co-exact attributions made from the diagram, complaints are in order" (Manders, 2008, p. 98), which might lead to reject concrete diagrams and re-draw new ones. The necessity of a diagram discipline in Euclid's diagram based geometric practice appears thus to be grounded, in part, in the cognitive difficulty of individuals to agree in judging co-exact relations in diagrams where such relations come close to exact ones.

\section{Conclusion}

The cognitive processing of spatial relations in Euclidean diagrams is an important issue both for the philosophy of mathematics with respect to the logical and epistemological analysis of how agents interact with diagrams in the context of Euclid's diagram-based geometric practice, and for cognitive science with regard to the analysis of the cognitive mechanisms at play in these interactions. In this study, we showed how the philosophical analysis of Euclid's diagram-based geometric practice due to Manders can be fruitfully combined with the experimental methodologies of the field of cognitive relation processing to move forwards on this issue: the philosophical analysis provides a conceptual framework for structuring the empirical inquiry of these interactions by singling out issues of particular importance and by providing essential conceptual distinctions; relevant experimental methodologies from the field of spatial relation processing can then be recruited to design experiments so as to yield empirical information on the cognitive mechanisms involved; the results thus obtained can in turn be shown to have various implications for the philosophical analysis itself. This study constitutes, however, only a preliminary attempt to foster interaction between philosophy and cognitive science on this 


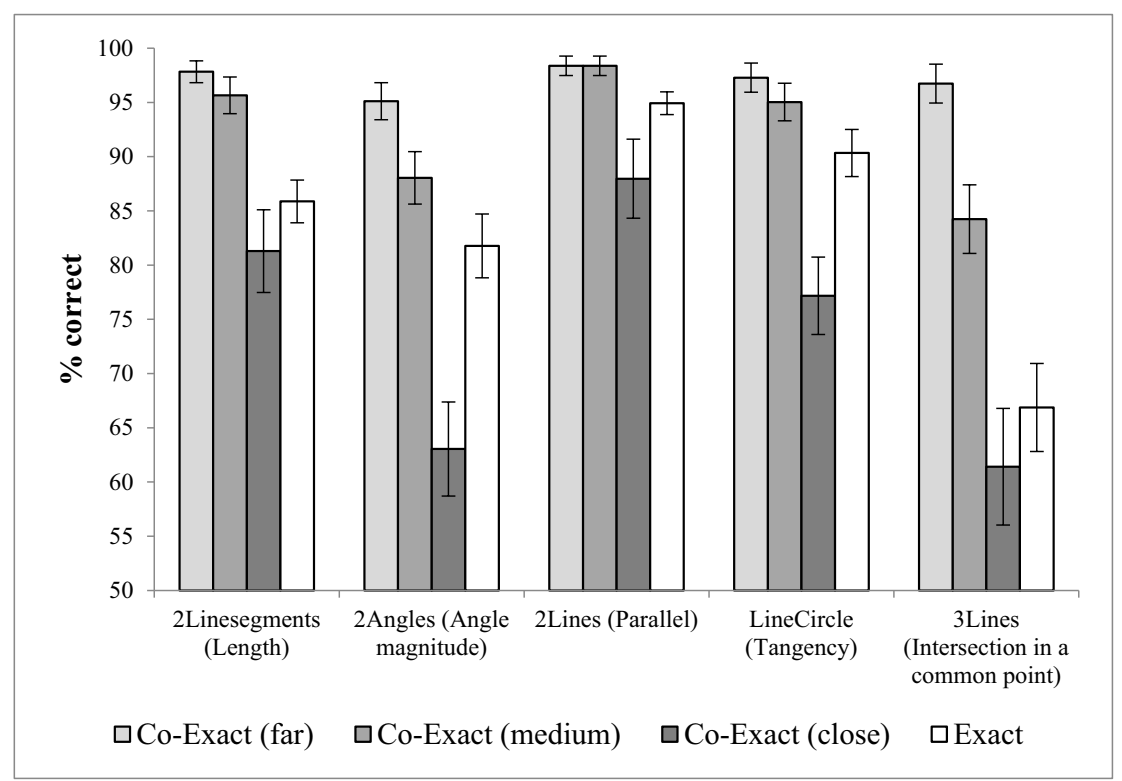

$\mathrm{a}$

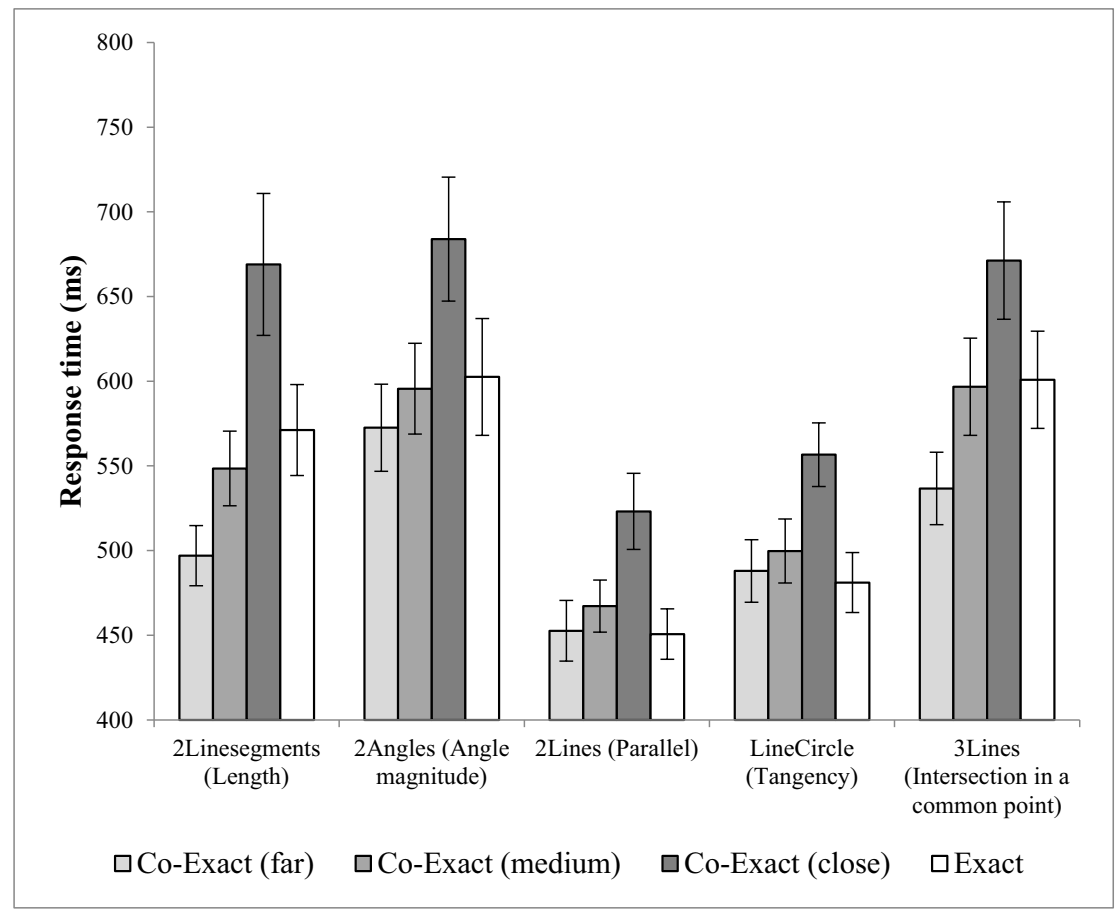

b

Fig. 5. a. Mean accuracy (\%) for exact and co-exact stimuli. Error bars represent Standard Error of the Mean. $b$. Mean response times (ms) for exact and co-exact stimuli. Error bars represent Standard Error of the Mean.

issue. Further interdisciplinary works in this direction may have the potential to yield important insights not only for the paradigmatic case of Euclid's diagram-based geometric practice, but also for other mathematical practices where diagrams play a substantial role.

\section{CRediT authorship contribution statement}

Yacin Hamami: Conceptualization, Investigation, Visualization, Writing - original draft. Milan N.A. van der Kuil: Conceptualization, Software, Investigation, Formal analysis, Visualization, Writing original draft. John Mumma: Conceptualization, Writing - review \& editing. Ineke J.M. van der Ham: Conceptualization, Formal analysis, Writing - original draft.

\section{Acknowledgements}

This study was partly funded from a Strategic Research Project (SRP) of the Vrije Universiteit Brussel (Free University Brussels). YH acknowledges support from a postdoctoral fellowship of the FWO (Research Foundation - Flanders). 


\section{References}

Bryant, D. J., \& Tversky, B. (1999). Mental representations of perspective and spatial relations from diagrams and models. Journal of Experimental Psychology: Learning, Memory, and Cognition, 25(1), 137-156.

Byrne, R. M., \& Johnson-Laird, P. (1989). Spatial reasoning. Journal of Memory and Language, 28(5), 564-575.

Cohn, A. G., \& Hazarika, S. M. (2001). Qualitative spatial representation and reasoning: An overview. Fundamenta Informaticae, 46(1-2), 1-29.

Coventry, K. R., \& Garrod, S. C. (2004). Saying, seeing, and acting: The psychological semantics of spatial prepositions. Hove: Psychology Press.

Egenhofer, M. J., \& Franzosa, R. D. (1991). Point-set topological spatial relations. International Journal of Geographical Information Systems, 5(2), 161-174.

Euclid (1959). Elements. New York: Dover Books.

Ferreirós, J. (2015). Mathematical knowledge and the interplay of practices. Princeton: Princeton University Press.

Franklin, N., Tversky, B., \& Coon, V. (1992). Switching points of view in spatial mental models. Memory \& Cognition, 20(5), 507-518.

Freeman, J. (1975). The modelling of spatial relations. Computer Graphics and Image Processing, 4(2), 156-171.

Giaquinto, M. (2007). Visual thinking in mathematics: An epistemological study. Oxford: Oxford University Press.

Hartshorne, R. (2000). Geometry: Euclid and beyond. New York: Springer-Verlag.

Hartshorne, R. (2000b). Teaching geometry according to Euclid. Notices of the American Mathematical Society, 47(4), 460-465.

Hayward, W. G., \& Tarr, M. J. (1995). Spatial language and spatial representation. Cognition, 55(1), 39-84.

Kelleher, J. D., \& Costello, F. J. (2009). Applying computational models of spatial prepositions to visually situated dialog. Computational Linguistics, 35(2), 271-306.

Knauff, M. (2013). Space to reason: A spatial theory of human thought. Cambridge, MA: MIT Press.

Koedinger, K. R., \& Anderson, J. R. (1990). Abstract planning and perceptual chunks: Elements of expertise in geometry. Cognitive Science, 14(4), 511-550.

Kosslyn, S., Koenig, O., Barrett, A., Backer Cave, C., Tang, J., \& Gabrieli, J. (1989). Evidence for two types of spatial representations: Hemispheric specialization for categorical and coordinate relations. Journal of Experimental Psychology: Human Perception and Performance, 15(4), 723-735.

Kosslyn, S. M. (1987). Seeing and imagining in the cerebral hemispheres: A computational approach. Psychological Review, 94(2), 148-175.

Kuipers, B. (1982). The "map in the head" metaphor. Environment and Behavior, 14(2), 202-220
Kuipers, B. (1983). The cognitive map: Could it have been any other way? In H. L. PickJr., \& L. P. Acredolo (Eds.). Spatial orientation: Theory, research, and application (pp. 345359). Boston, MA: Springer.

Landau, B., \& Jackendoff, R. (1993). "What" and "where" in spatial language and spatial cognition. Behavioral and Brain Sciences, 16(2), 217-238.

Landy, D., \& Goldstone, R. L. (2007). How abstract is symbolic thought. Journal of Experimental Psychology: Learning, Memory, and Cognition, 33(4), 720-733.

Landy, D., \& Goldstone, R. L. (2010). Proximity and precedence in arithmetic. Quarterly Journal of Experimental Psychology, 63(10), 1953-1968.

Mancosu, P. (2008). The philosophy of mathematical practice. Oxford: Oxford University Press.

Manders, K. (2008). The Euclidean diagram. In P. Mancosu (Ed.). The philosophy of mathematical practice (pp. 80-133). Oxford: Oxford University Press.

McDonough, L., Choi, S., \& Mandler, J. M. (2003). Understanding spatial relations: Flexible infants, lexical adults. Cognitive Psychology, 46(3), 229-259.

McNamara, T. P. (1986). Mental representations of spatial relations. Cognitive Psychology, 18(1), 87-121.

Mueller, I. (1981). Philosophy of mathematics and deductive structure in Euclid's Elements. Cambridge MA: The MIT Press.

Munnich, E., Landau, B., \& Dosher, B. A. (2001). Spatial language and spatial representation: A cross-linguistic comparison. Cognition, 81(3), 171-208.

Netz, R. (1999). The shaping of deduction in Greek mathematics. Cambridge: Cambridge University Press.

Randell, D. A., Cui, Z., \& Cohn, A. G. (1992). A spatial logic based on regions and connection. Proceedings of the third international conference on knowledge representation and reasoning (pp. 165-176). San Mateo, CA: Morgan Kaufmann.

Retz-Schmidt, G. (1988). Various views on spatial prepositions. AI Magazine, 9(2), 95-105.

Stevens, A., \& Coupe, P. (1978). Distortions in judged spatial relations. Cognitive Psychology, 10(4), 422-437.

Tversky, B. (1993). Cognitive maps, cognitive collages, and spatial mental models. COSIT 1993. Lecture notes in computer science: . Vol. 716. Spatial information theory a theoretical basis for GIS (pp. 14-24). Berlin: Springer.

van der Ham, I., Postma, A., \& Laeng, B. (2014). Lateralized perception: The role of attention in spatial relation processing. Neuroscience and Biobehavioral Reviews, 45, $142-148$.

Winston, M. E., Chaffin, R., \& Herrmann, D. (1987). A taxonomy of part-whole relations. Cognitive Science, 11(4), 417-444.

Winston, P. H. (1970). Learning structural descriptions from examples. AI Technical Report AITR-231, TR-76MIT 